\title{
A Compactness based Saliency Approach for Leakages Detection in Fluorescein Angiogram
}

\author{
Yitian Zhao - Pan Su • Jian Yang • \\ Yifan Zhao - Yalin Zheng • Yongtian \\ Wang
}

Received: date / Accepted: date

\begin{abstract}
This study has developed a novel saliency detection method based on compactness feature for detecting three common types of leakage in retinal fluorescein angiogram: large focal, punctate focal, and vessel segment leakage. Leakage from retinal vessels occurs in a wide range of retinal diseases, such as diabetic maculopathy and paediatric malarial retinopathy. The proposed framework consists of three major steps: saliency detection, saliency refinement and leakage detection. First, the Retinex theory is adapted to address the illumination inhomogeneity problem. Then two saliency cues, intensity and compactness, are proposed for the estimation of the saliency map of each individual superpixel at each level. The saliency maps at different levels over the same cues are fused using an averaging operator. Finally, the leaking sites can be detected by masking the vessel and optic disc regions. The effectiveness of this framework has been evaluated by applying it to different types of leakage images with cerebral malaria. The sensitivity in detecting large focal, punctate focal and vessel segment leakage is $98.1 \%, 88.2 \%$ and $82.7 \%$, respectively, when compared to a reference standard of manual annotations by expert human observers. The developed framework will become a new powerful tool for studying retinal conditions involving retinal leakage.
\end{abstract}

Yitian Zhao, Jian Yang, and Yongtian Wang

Beijing Engineering Research Center of Mixed Reality and Advanced Display, School of Optics and Electronics, Beijing Institute of Technology, Beijing, China

E-mail: yitian.zhao@bit.edu.cn

$\mathrm{Pan} \mathrm{Su}$

School of Control and Computer Engineering, North China Electric Power University, Baiding, 071003, China

Yifan Zhao

BEPSRC Centre for Innovative Manufacturing in Through-life Engineering Services, Cranfield University, Cranfield, UK

Yalin Zheng

Department of Eye and Vision Science, University of Liverpool, Liverpool, UK 
Keywords Saliency - Compactness - Leakage · Fluorescein angiography · Malarial retinopathy

\section{Introduction}

Fluorescein angiography (FA) is derived by taking a series of digital photographs of the retina before and after the fluorescein reaches the retinal circulation. FA's ability to capture a range of retinal abnormalities in differential diagnosis of retinal diseases, such as age-related macular degeneration (AMD), diabetic retinopathy (DR) and malarial retinopathy (MR) [1-3]. FA provides a map of retinal vascular structure and function by highlighting blockage to, and leakage from, retinal vessels.

Retinal vessel leakage is particularly relevant to cerebral malaria (CM) $[2$, 3]. Three types of leakage can be observed on fluorescein angiogram (FA) of malaria retinopathy (MR): large focal, punctate focal, and vessel segment leakage [4], see Fig. 1. Large focal leakage describes one or more large, usually circular, areas of leak, where the greatest linear diameter is larger than $125 \mu \mathrm{m}$. Punctate focal leak involves small but intensely bright sites of leak, where the greatest linear diameter is less than $125 \mu \mathrm{m}$. Leakage from vessel segments appears as increased brightness and blurring of vessels. These three types of leakage on MR recently have been defined on [5].
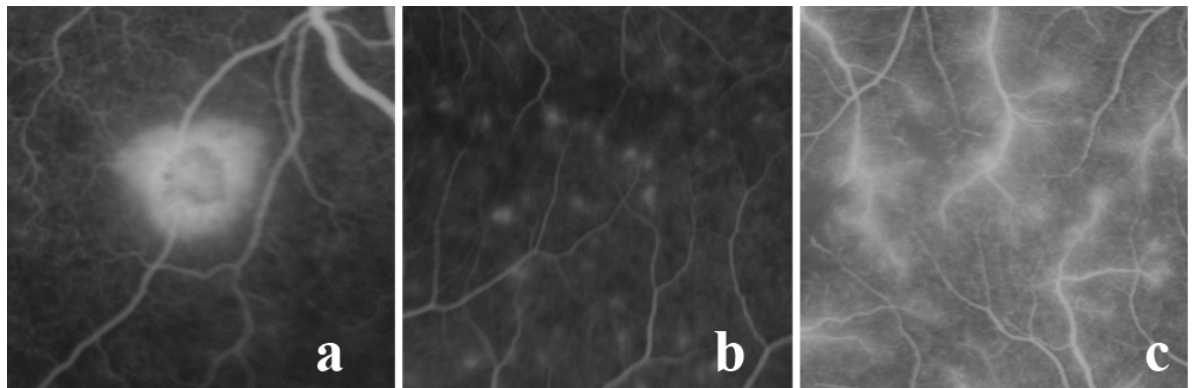

Fig. 1 Illustration of three types of leakage: (a) Large focal leakage. (b) Punctate focal leakage. (c) Vessel segment leakage.

However, the research of detecting leakage on retinal image is relatively unexplored. To the best of our knowledge, there is no automated method to detect leakage on MR. In other ocular diseases has received little, such as diabetic retinopathy [6], retinal vein occlusion [7], hyperfluorescent[8] and choroidal neovascularization [9-11], if any, attention. In the section, only the related works on detecting the abnormalities on different modalities of medical images by saliency information will be reviewed. Yuan et al. [12] proposed a saliency based ulcer detection method for wireless capsule endoscopy (WCE) diagnosis. It uses the multi-level superpixel representation as the pre-processing of saliency detection, and the saliency map is generated by a fusion strategy of 
integrating all obtained saliency maps from all levels. This method is capable to represent the accurate contour of the ulcer regions, and the ulcer regions are located by classification tasks. Mahapatra and Sun used the saliency and gradient information in Markov random field for non-rigid registration of dynamic MR cardiac perfusion images [13]. This approach addresses the problem that majority of the nonrigid registration algorithms do not give satisfactory results in the presence of intensity changes. A visual saliency based bright lesion detection is introduced in [14]. The spectral residual saliency model [15] was employed to compute the saliency map of the color fundus retinal images. The saliency computation obtains a sparse representation of images, and an image can be classified as normal or abnormal (having bright lesions) by the obtained saliency information. Jampani et al. [16] analyzed the relevance of saliency models in detecting abnormalities in two types of medical images. The authors extended the graph based visual saliency [17] models to detect the diffuse lesions in chest X-ray images, and high contrast lesions in retinal images. Zhao et al. [18] proposed a framework to detect the vessel abnormalities on FA with application to malarial retinopathy. The authors used the intensity and shape information to generate the saliency map to detect the intra vascular filling defect. However, the domain of uncertainty-based image processing techniques may also be employed to yield the abnormality detection [19-24].

The limitation of these works are that the effectiveness and robustness is not convincing enough, such as the dataset used for validation in [12] is relative too small. The saliency provided a high quality of contrast enhanced images by [13], but the gradient information still can be influenced by noise and does not accurately register the boundary of the left ventricle. The approach [14] is invalid on detection spot lesions, such as microneurysms.

\subsection{Proposed Work}

In this application, we define saliency in terms of information content: a keypoint corresponds to a particular image location within a structure with a low probability of occurrence (i.e. high information content). For example, leakage of fluorescent dye causes a large difference in brightness between the leak and surrounding non-leaking areas. In this instance, the leaking regions may be defined as salient regions as the leakage of fluorescent dye causes a large difference in brightness between the leak and surrounding non-leaking areas. We have successfully integrated the intensity and compactness information to generate the saliency map for the detection of leakage in FA image. By gathering the intensity and compactness features, the estimated saliency map is not only able to distinguish the regions where the intensities are significant different to their surroundings, but also has the capacity to avoid the nonleaking regions with large intensity by considering the compactness of the objects in the given images. The contributions of the proposed method can be concluded as follows: 
First, we propose a new unsupervised technique to detect and quantify the type of leakage in MR by a novel adaptation of the concept of saliency [25]. Saliency is a predictor of object regions which attract human attention. Saliency emerges from such characteristics in features of the image as visual uniqueness, unpredictability, or rarity, and is often attributed to variations in specific image attributes such as color, gradient, edges, and boundaries [26,27]. Such attributes are characteristic of retinal leakage in FA images. It indicates the relative importance of visual features, and is closely related to the characteristics of human perception and processing of visual stimuli $[25,28]$. The most general model of saliency detection is described by Itti and Koch [25]. Other existing saliency detection methods for feature determination can be divided into four classes: pixel-based methods [29-31,25,32,17,33]; regionbased methods [26,34,28]; frequency-based methods [15,35-37]; parameter learning-based methods [38-40].

Second, we have proposed a new feature - compactness to refine the intensitybased saliency map. Normally, human observers pay more attention to a more compact object than to a more diffuse object. The measure of compactness of an object might therefore be of use as a complementary feature to intensity for saliency measurement, with the aim of reducing the number of falsely-detected salient regions.

The proposed leakage detection framework consists three main phases: saliency estimation, saliency refinement, and leakage detection. The performance of this framework also will be evaluated against the human expert reference standard. The rest of this paper is organized as follows: section 2 describes the leakage detection methods, which consists of saliency detection and refinement. A brief introduction to the dataset and evaluation metrics are provided in section 3. In addition, the experimental results and the evaluations against the human graders on detecting leakage are also demonstrated in section 3. Finally, the paper is discussed and concluded in section 4.

\section{Method}

The proposed framework consists of three main phases, which are saliency detection, saliency refinement, and leakage detection. In this section, all procedures are described in detail.

\subsection{Saliency Detection}

We now describe our saliency computation, which is based on the assumptions that (i) a salient region is always different from its surrounding context [40], and (ii) a more compact object tends to draw more visual attention [41]. The proposed saliency detection is formulated from Shannon's information definition. In the case of images, the content of a region around one pixel would be more informative than a single pixel. Let $\mathcal{P}_{i} \in I$ be the viable local 


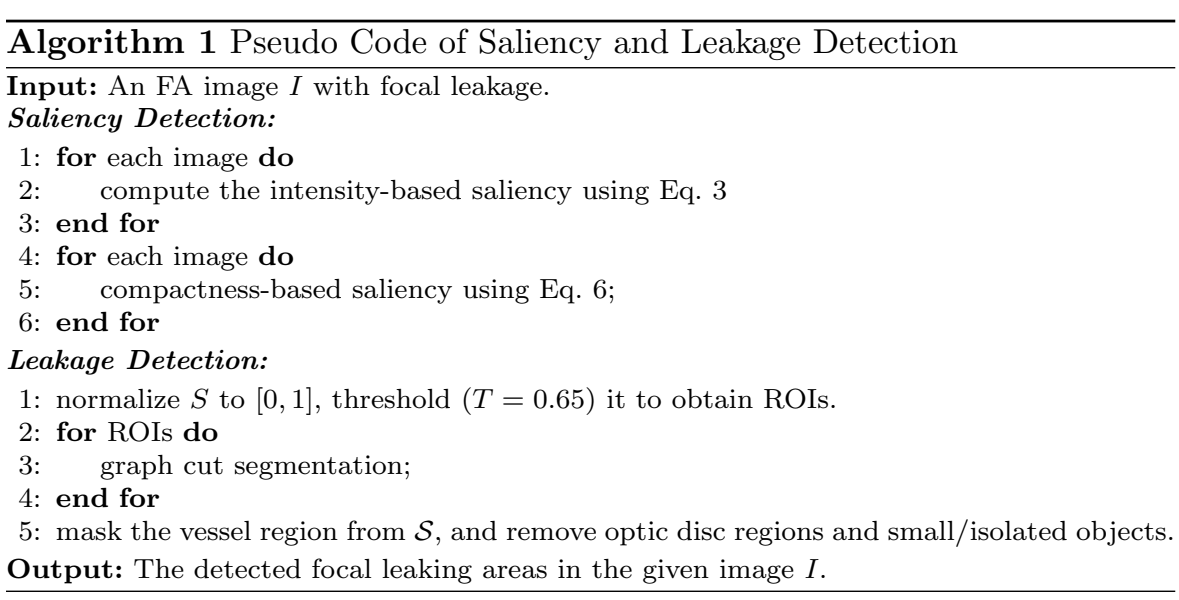

representation as a patch that represents pixel $i$ (here, a $3 \times 3$ window centred on pixel $i$ is used to define the patch), and $I$ indicates the input image. The patches can be seen as samples of a multivariate probability function (PDF). The kernel density estimator (KDE) is chosen, as, being non-parametric, it will permit the estimation of any $\mathrm{PDF}$. The probability of a patch $\mathcal{P}_{j}$ may now be defined as

$$
p\left(\mathcal{P}_{j}\right)=\frac{1}{N h} \sum_{i \in I} K\left(\frac{d\left(\mathcal{P}_{j}, \mathcal{P}_{i}\right)}{h}\right),
$$

where $d$ is a distance function that will be discussed later, $K$ is a kernel, $h$ is a smoothing parameter, and $N$ represents the number of pixels. The KDE method has the capacity to average out the contribution of each sample $i$ by spreading it over a certain area [42], which is defined by $K$. The multivariate distribution will have a higher probability if the patches are in dense areas. From our experience, the most commonly used and appropriate kernel is a Gaussian function with zero mean and standard deviation $\sigma_{k}$. Using a Gaussian kernel, equation (1) can be rewritten as

$$
p^{\prime}\left(\mathcal{P}_{j}\right)=\frac{1}{N \Gamma} \sum_{i \in I} e^{\frac{d^{2}\left(\mathcal{P}_{j}, \mathcal{P}_{i}\right)}{2 \sigma_{k}^{2}}} .
$$

The estimated probabilities are taken from an actual PDF by setting a proper constant $\Gamma . \sigma=0.2$ is chosen to substitute for $h$. After determining the probability of the patches, the intensity-based saliency $\mathrm{S}$ measure can be defined as follows:

$$
\mathrm{S}(j)=-\log \left(\frac{1}{N \Gamma} \sum_{i \in I} e^{-\left(\frac{d^{2}\left(\mathcal{P}_{j}, \mathcal{P}_{i}\right)}{2 \sigma_{k}^{2}}\right)}\right),
$$

where $d$ is relative average distance. The relative distance is used in case the distribution of the data is not uniform, and the distance metric mainly focuses 


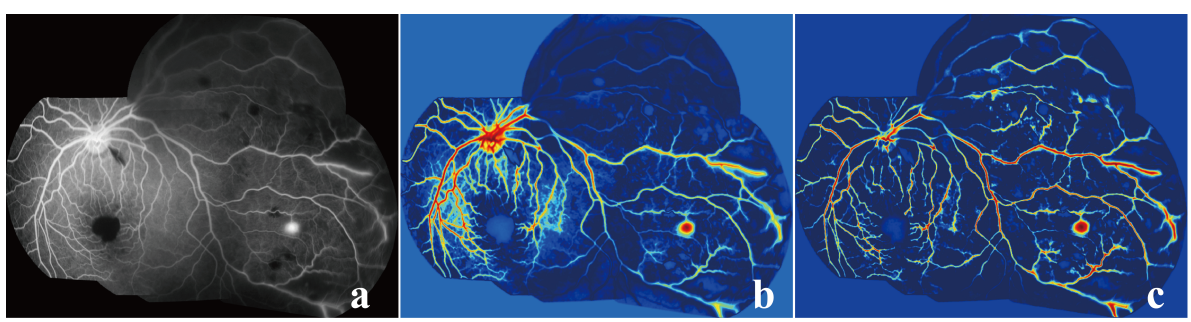

Fig. 2 Illustration of two saliency maps derived from the same FA image. (a) The example FA image. (b) A saliency map based on intensity only; (c) A saliency map based on intensity enhanced by compactness measure.

on the relationships between neighboring points. Let a patch set $W$ in the input image contains $n$ patches $\mathcal{P}_{1}, \mathcal{P}_{2}, \ldots \mathcal{P}_{n}$. The relative average distance of a pair of patches $\mathcal{P}_{i}, \mathcal{P}_{j} \in W$ is defined as follows:

$$
d\left(\mathcal{P}_{j}, \mathcal{P}_{i}\right)=\frac{\left\|\mathcal{P}_{i}-\mathcal{P}_{j}\right\|_{2}}{\left.{\text { ave } \mathcal{P}_{k} \subset W} \subset \mathcal{P}_{i}-\mathcal{P}_{k} \|_{2}\right)} .
$$

The ave $\mathcal{P}_{k} \subset W\left(\left\|\mathcal{P}_{i}-\mathcal{P}_{k}\right\|_{2}\right)$ are the average Euclidean distance between $\mathcal{P}_{i}$ and other patches $\mathcal{P}_{k}$ belonging to $W$ respectively.

For two sets of points/pixels with similar neighboring relationships but different densities (i.e., similar relative density), the absolute distances between corresponding points differ dramatically from each other, but the relative distances are in general similar [43]. This is an advantage of the relative distance metric in reflecting the relative density of points and relative scale of the imaged objects. In this application, the intensity-based saliency will finally be normalized into range $[0,1]$.

\subsection{Saliency Refinement}

Intuitively, the leakage region in a FA image presents different intensity information compared with the normal FA cases. However, it is observed that using the intensity feature alone to detect the salient region is not always successful [26], also has been evidenced by Fig. 2(b). The non-vessel regions with high-brightness in the middle of the image are also assigned as high salient, but we perceive the leakage and vessels as more salient. Therefore, this section introduces an additional feature - compactness. Normally, human pay more attention on a more compact object than a more diffuse object. The measure of compactness of an object might be used as a complementary feature for saliency measurement, with the aim of overcoming the conflict of the falsely detected salient region.

For patch $\mathcal{P}_{j}$, the compactness $c\left(\mathcal{P}_{j}\right)$ is defined as

$$
c\left(\mathcal{P}_{j}\right)=\exp \left(-\alpha \frac{\sigma_{x, j}+\sigma_{y, j}}{\sqrt{\mathrm{X}^{2}+\mathrm{Y}^{2}}}\right)
$$




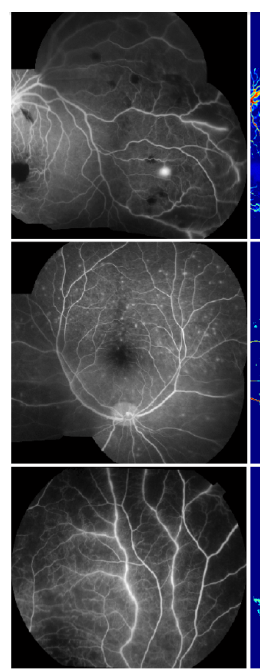

$\mathbf{a}$
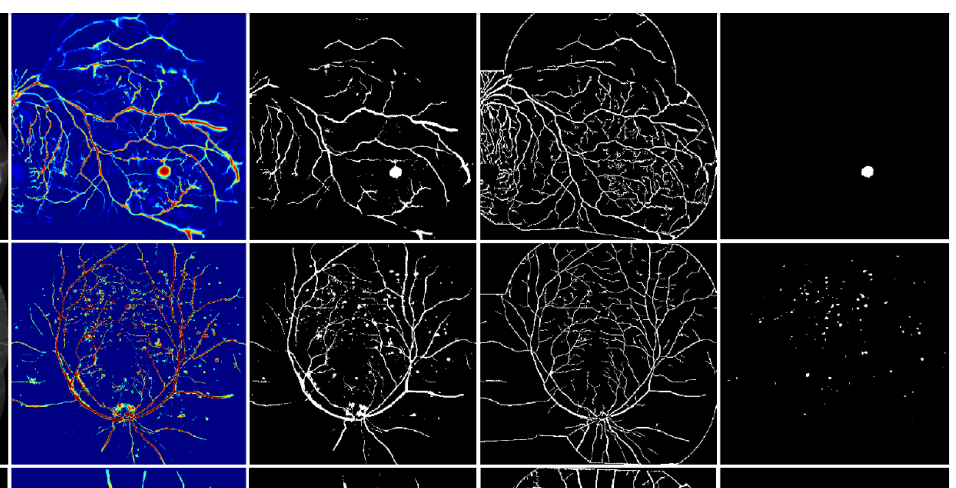

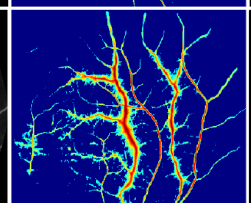

b

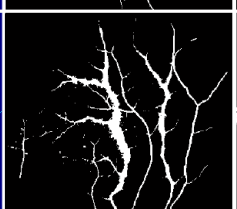

c

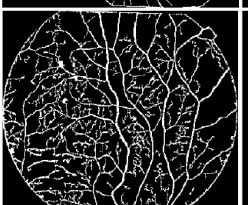

d

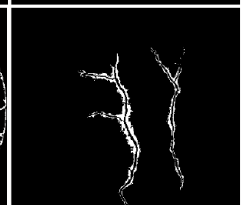

$\mathbf{e}$

Fig. 3 Overview of the main steps taken by our algorithm for detecting three type of leakages. From top to bottom: large focal leakage, punctate leakage, and vessel segment leakage. (a) Example FA images. (b) Saliency maps of (a). The warmer color indicates the more salient regions, and the cooler color shows the less salient regions. (c) Binary images of (b). (d) Vessel segmentation results. (e) The detected leakage regions.

where $\sigma_{x, q}$ and $\sigma_{y, q}$ are the standard deviations of the $x$ and $y$ coordinates of centroid of patch in $\mathcal{P}_{j}$, and $\alpha$ is a constant factor that is empirically set to 15 . $\mathrm{X}$ and $\mathrm{Y}$ are the width and height of the input image. Then by incorporating the compactness information with intensity of given image, the compactnesswise dissimilarity measure between $\mathcal{P}_{i}$ and $\mathcal{P}_{j}$ can be defined as

$$
\begin{aligned}
\operatorname{dis}_{\text {compact }}\left(\mathcal{P}_{i}, \mathcal{P}_{j}\right)=\left\|\mathrm{S}_{i}-\mathrm{S}_{j}\right\| & \times\left(1+\frac{c\left(\mathcal{P}_{j}\right)-c\left(\mathcal{P}_{i}\right)}{2}\right) \\
& \times \exp \left(-\frac{\beta d\left(\mathcal{P}_{j}, \mathcal{P}_{i}\right)}{\sqrt{\mathrm{X}^{2}+\mathrm{Y}^{2}}}\right),
\end{aligned}
$$

where term $\left\|\mathrm{S}_{i}-\mathrm{S}_{j}\right\|$ indicates the distinctiveness of intensity-based characteristic of patch $\mathcal{P}_{i}$ and $\mathcal{P}_{j} . d\left(\mathcal{P}_{j}, \mathcal{P}_{i}\right)$ is the relative average distance of patch $\mathcal{P}_{j}$ and $\mathcal{P}_{j}$, which has been proposed in Eq (3). The constant factor $\beta$ is empirically set to 300 . The compactness-based saliency of $\mathcal{P}_{j}$ can be written as

$$
\mathrm{S}^{\prime}\left(\mathcal{P}_{j}\right)=1-\exp \left(-\sum_{j=1, j \neq i}^{n} \operatorname{dis}_{\text {compact }}\left(\mathcal{P}_{i}, \mathcal{P}_{j}\right)\right)
$$

Fig. 2 demonstrates the effectiveness of the saliency refinement. It can be observed the region of interest, such as large vessel and leaking areas, have been highlighted, and the background is suppressed. 


\subsection{Leakage Detection}

The overview of the main steps taken by our algorithm for detecting three type of leakages have been demonstrated on Fig. 3. After using the proposed saliency detction method, the contrast between vessels/leakages and background has been successfully enhanced (Fig. 3(b)). The appearance of these leaks are highlighted, and the background regions are suppressed, when compared to the original image.

Once the saliency map is computed and normalized to $[0,1]$, a threshold value $T=0.65$ is applied to the saliency map to obtain the ROIs, as shown as Fig. 3(c). The most common methods to detect the leaking region by thresholding approach. However, the thresholding approach cannot guarantee smooth boundaries of the structure, and always generate isolated fragments. In light of this inadequacy, more sophisticated segmentation would perform better results. On the other hand, the computation cost of a segmentation tool is also an important factor to be taken into account for potential real applications. For these two reasons, we advocate here a graph cut based segmentation model[44] on the obtained ROIs to locate the leakage.

Let $\mathcal{N}$ be the set of edges $\{(u, v)\}$, and $\mathcal{M}$ denote the number of image pixels, the discrete energy function can be given as:

$$
E(x)=\sum_{u=1}^{m} E_{u}\left(x_{u}\right)+\sum_{\{u, v\} \in \mathcal{N}} E_{u v}\left(x_{u}, x_{v}\right),
$$

where $x=\left(x_{1}, \cdots, x_{\mathcal{N}}\right)$ is the binary labelling where the $x_{u}$ is either 0 or 1 depending on whether the pixel $u$ belongs to background $\Omega_{1}$ or foreground $\Omega_{2}$. The first term here approximates the region terms while the second term approximates the regularization term. The unary term $E_{u}$ and binary term $E_{u v}$ are defined as:

$$
\begin{gathered}
E_{u}^{0}=\lambda_{1}\left(I_{0 u}-c_{1}\right)^{2}, E_{u}^{1}=\lambda_{2}\left(I_{0 u}-c_{2}\right)^{2} \\
E_{u, v}= \begin{cases}\mu w_{u v}, & \text { if } x_{u} \neq x_{v} \\
0, & \text { otherwise. }\end{cases}
\end{gathered}
$$

where $E_{u}^{0}, E_{u}^{1}$ denote the weights between the node $u$ and the two terminals while $w_{u v}$ denotes the weight between neighboring pixels $u$ and $v$.

The infinite perimeter active contour with hybrid region (IPACHR) method [45] is used to segment retinal vessels for its good performance.In brief, this method uses an infinite perimeter active contour model for its effectiveness in detecting objects (e.g. vessels) with irregular and oscillatory boundaries. Moreover, this method considers hybrid region information (local phase based vesselness map and intensity) in an image in order to achieve further improved performance compared to the standard infinite perimeter active contour model. For more details, we refer readers to the original paper [45].

Any small and/or isolated objects are eliminated after masking the vessel regions, by the use of a disk-shaped opening operation with a radius of 2 
pixels. In most cases, the optic disc is detected as a salient region, and should be removed. In this framework, the optic disc can be distinguished by counting the number of nearby vessels in the remaining regions. Typically, the number of vessels surrounding the optic disc is much larger than the number of vessels close to large focal leaking sites. The threshold vessel number is empirically chosen as 5 . In other words, a region with a surrounding vessel number greater than 5 will be assumed to be the optic disc $[46,18]$, and will be removed. In our experiments this method is found to be efficient and effective, however, the other optic disc removal methods may equally be used. Finally, the retained regions could be defined as the leaking area Fig. 3(e).

\section{Experimental Results}

The detection framework in three types of leakage was implemented in Matlab 2013a. All the experiments were performed on a PC with Intel(R) Core(TM)i7$4790 \mathrm{~K} \mathrm{CPU} 4.0 \mathrm{GHz}$ system and 16GB RAM.

\subsection{Dataset}

The dataset we used comprises retinal FA images taken from children with CM admitted to the Malaria Research Project Ward, Queen Elizabeth Central Hospital, Blantyre, Malawi. Ethical approval for retinal examination and imaging was given by committees in Blantyre and at collaborating institutions. Consent was given by the guardians of subjects before examination and imaging. The images were systematically sorted and graded for quality by the Reading Centre at St Paul's Eye Unit, Royal Liverpool University Hospital and Department of Eye and Vision Science, University of Liverpool. For more details on this dataset, we refer to [5].

\subsection{Results and Discussions}

In the large focal and punctate leakage detections, the montage images were used, to avoid over-counting sites of leakage. 20 images (one per patient) with large focal leakage, and 10 images from 6 patients with punctate leakage were used. Note, punctate focal leakage is relatively less common compared with the other two leakage types (large focal and vessel segment leakage), only 6 patients were found with this leakage in database, and 10 images from these patients (including both macula-centred and montage images) were chosen for evaluation. An ophthalmologist (IJCM) defined the boundaries of each large focal leak, and the centre point of each punctate focal leak. The sensitivity, false positive per image, false negative per image, and ratio of overlapping area (OR) were used as the evaluation metrics. The OR is defined as:

$$
\mathrm{OR}=\frac{\text { \#the pixel length of overlapped region }}{\# \text { the pixel length of the annotated leak area by human expert }} .
$$




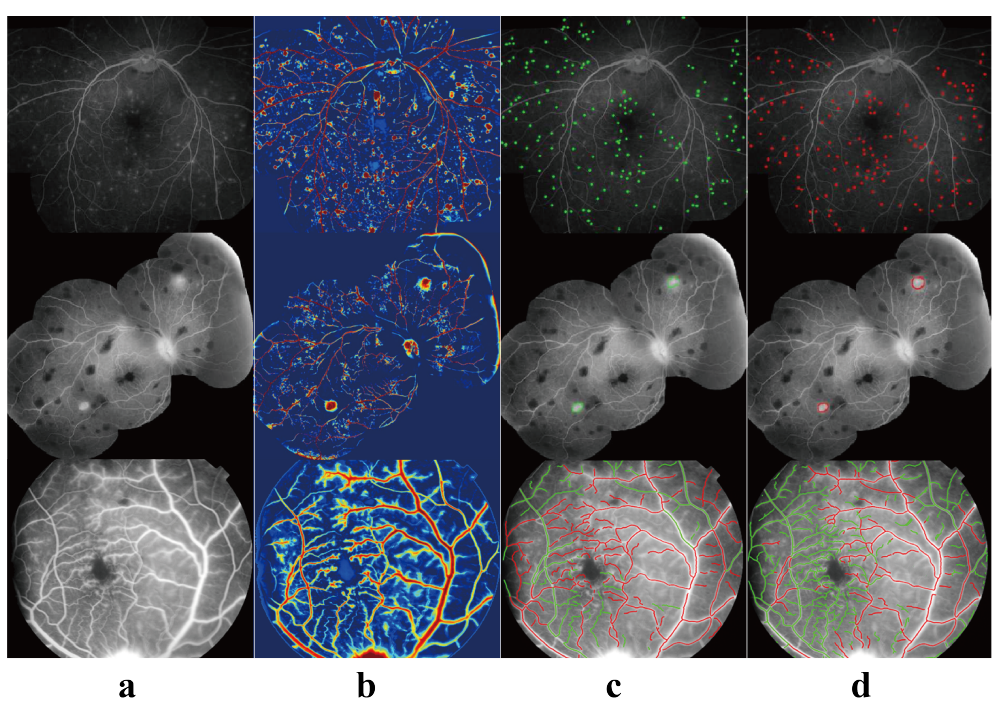

Fig. 4 Detection of three types of leakage by our automated method and a human observer. (a) Input FA images with leakage. (b) Saliency maps of (a), most of the vessels and leaking regions have been highlighted with 'warmer' color. (c) Manual annotations. (d)Automated detection by our method. In the case of vessel segment leakage detection, the leaking vessels are assigned as red, while normal vessels as green.

\subsubsection{Punctate focal}

The top row of Fig. 4 show that the punctate focal leak regions were detected by both the human expert and the proposed method, respectively. It can be seen that most sites of punctate focal leaking region were located by our automated framework. Table 1 illustrates the evaluation results of our automated method on detecting punctate focal leakage, in terms of sensitivity, false positives (FP) per image of punctate focal leakage number, and the false negatives $(\mathrm{FN})$ per image of punctate focal leakage number. According to the human reference standard there were 240 sites of punctate focal leakage in the tested 10 images. The sensitivity of the detection on punctate focal leakage is 0.882 , which shows the proposed method has successfully detected 211 out of these 240 sites of leakage. However, 29 site were missed, leading to a false negative ratio of 2.832 per image. Meanwhile, false positive ratio is 1.1 per image, because 11 sites were falsely determined as punctate focal leakage by proposed method due to the image artefact and local imbalanced illumination.

\subsubsection{Large focal}

The middle row of Fig. 4 demonstrates the detection of large focal leakage by both human expert and the proposed method, respectively. It can be seen that the detected leakage regions are slightly different, since it is difficult to exactly define the boundary of a leaking area by hand since the contrast gradually 
Table 1 The performance of the proposed framework on detecting large focal and punctate focal leakge.

\begin{tabular}{|l|c|c|}
\hline & punctate focal & large focal \\
\hline sensitivity of leakage detection & 0.882 & 0.981 \\
\hline FP number of leakage per image & 1.100 & 0 \\
\hline FN number of leakage per image & 2.832 & 0.050 \\
\hline ratio of overlapping area & - & 0.891 \\
\hline
\end{tabular}

fades at the edge of the lesion. Table 1 also indicates the evaluation results of the developed framework in the detection of large focal leakage, in terms of sensitivity, false positives of focal leakage regions per image, false negatives per image of focal leakage regions, and the overlapping area between the regions detected by the proposed method and human expert. In these studied images, a total of 41 sites of large focal leak were identified by the human expert reference standard. In our framework, it only failed to detect 1 out of these focal leak sites, which has been evidenced by the sensitivity ratio of 0.976 , and has a false negative ratio of 0.05 per image. Meanwhile, our method has a false positive ratio of 0 , which means there were no regions falsely identified as large focal leakage.

\subsubsection{Vessel segment}

The bottom of Fig. 4 indicate the results of vessel segment leakage detection. Green designates the non-leaking vessels and red the leaking vessels. It is worth noting that only vessels labeled by human observers were considered for detection. In contrast, the second human observer appears to over-detect abnormal vessels. It clearly can be seen that the results of our automated method and the reference standard are very similar. Table 2 shows the evaluation results of the proposed vessel segment leakage detection in terms of sensitivity (Se), specificity (Sp), accuracy (Acc), and area under curve (AUC). These metrics are defined as follows:

$$
\begin{aligned}
& \mathrm{Se}=\frac{\mathrm{tp}}{\mathrm{tp}+\mathrm{fn}}, \\
& \mathrm{Sp}=\frac{\mathrm{tn}}{\mathrm{tn}+\mathrm{fp}}, \\
& \mathrm{Acc}=\frac{\mathrm{tp}+\mathrm{tn}}{\mathrm{tp}+\mathrm{fp}+\mathrm{tn}+\mathrm{fn}}, \\
& \mathrm{AUC}=\frac{\mathrm{Se}+\mathrm{Sp}}{2},
\end{aligned}
$$

where tp, tn, fp and fn indicate the true positive (the number of correctly identified leaking vessel segments), true negative (the number of correctly 
Table 2 Quantitative analysis of the performance of the proposed method on vessel segments leakage detection and manual grading of the leakage vessel.

\begin{tabular}{|c|llll|llll|}
\hline \multirow{2}{*}{ Data } & \multicolumn{4}{|c}{ Automatic vs. Manual } & \multicolumn{4}{c|}{ Manual Inter-observer } \\
\cline { 2 - 9 } & Se & Sp & Acc & AUC & Se & Sp & Acc & AUC \\
\hline $\mathbf{1}$ & 0.792 & 0.855 & 0.781 & 0.823 & 0.813 & 0.726 & 0.802 & 0.770 \\
\hline $\mathbf{2}$ & 0.864 & 0.786 & 0.816 & 0.825 & 0.754 & 0.372 & 0.652 & 0.563 \\
\hline $\mathbf{3}$ & 0.830 & 0.846 & 0.819 & 0.838 & 0.812 & 0.512 & 0.688 & 0.662 \\
\hline $\mathbf{4}$ & 0.921 & 0.803 & 0.876 & 0.862 & 0.946 & 0.956 & 0.946 & 0.951 \\
\hline $\mathbf{5}$ & 0.629 & 0.764 & 0.715 & 0.697 & 0.810 & 0.895 & 0.864 & 0.852 \\
\hline $\mathbf{6}$ & 0.849 & 0.842 & 0.811 & 0.845 & 0.740 & 0.881 & 0.724 & 0.810 \\
\hline $\mathbf{7}$ & 0.884 & 0.829 & 0.831 & 0.856 & 0.774 & 0.786 & 0.736 & 0.780 \\
\hline $\mathbf{8}$ & 0.797 & 0.850 & 0.794 & 0.823 & 0.783 & 0.743 & 0.738 & 0.763 \\
\hline $\mathbf{9}$ & 0.829 & 0.878 & 0.817 & 0.854 & 0.773 & 0.879 & 0.745 & 0.826 \\
\hline $\mathbf{1 0}$ & 0.873 & 0.884 & 0.861 & 0.878 & 0.726 & 0.612 & 0.680 & 0.669 \\
\hline Mean \pm & $\mathbf{0 . 8 2 7} \pm$ & $\mathbf{0 . 8 3 4} \pm$ & $\mathbf{0 . 8 1 2} \pm$ & $\mathbf{0 . 8 3 0} \pm$ & $\mathbf{0 . 7 9 3} \pm$ & $\mathbf{0 . 7 3 6} \pm$ & $\mathbf{0 . 7 5 8} \pm$ & $\mathbf{0 . 7 6 5} \pm$ \\
Std. & $\mathbf{0 . 0 8 0}$ & $\mathbf{0 . 0 3 9}$ & $\mathbf{0 . 0 4 4}$ & $\mathbf{0 . 0 5 0}$ & $\mathbf{0 . 0 6 2}$ & $\mathbf{0 . 1 8 7}$ & $\mathbf{0 . 0 9 0}$ & $\mathbf{0 . 1 1 0}$ \\
\hline
\end{tabular}

identified non-leaking vessel segments), false positive (the number of incorrectly identified leaking vessel segments), and false negative(the number or incorrectly identified non-leaking vessel segments), respectively. In particular $A U C$ is calculated as suggested by Hong et al [47]. An AUC of 1.0 means that the classifier distinguishes class examples perfectly.

Two human experts were asked to selected leaking vessels and non-leaking vessels independently, and a consensus annotation between the two of them was used as the final reference standard. In order to assess inter-observer variation, a third grader labeled the vessels using the same method. According to the human reference standard these 10 images contained 2,103 leaking vessel segments. Compared to the reference standard, the sensitivity, specificity, accuracy, and AUC of the automated method is $0.827 \pm 0.08,0.834 \pm 0.039$, and $0.812 \pm 0.044$. The previously reported degree of correspondence between the third human observer's annotation and the reference annotation are also provided. It is noteworthy that the interobserver sensitivity, specificity, and accuracy were lower than our automatic method: $0.793 \pm 0.062,0.736 \pm 0.736$ and $0.758 \pm 0.09$, respectively. We also considered the area under the curve (AUC), which is equal to 1 for a perfect system, as a single metric to quantify the performance of our framework in detecting vessel segments leakage. In contrast, the AUC rate of automated method is 0.065 higher than human observer. In brief, this table shows that the developed automatic vessel segments leakage detection method can perform better than or at least as well as a human expert.

\section{Conclusions}

This paper proposed an innovative framework for the detection of three types of leakage founded on saliency detection, with the aim of supporting the study 
of abnormalities revealed in retinal images. The framework benefits from three major components: saliency estimation, saliency refinement, and leakage detection. We have successfully integrated compactness based feature to refined the intensity based saliency map. The proposed saliency detection method is promising on generating accurate saliency maps with well-highlighted leaking area, and the obtained saliency map is capable to guide the detection of leakage. The effectiveness of this method has been tested on FA images from patients with malarial retinopathy.

Our method demonstrated satisfactory overall performance on large focal, punctate focal and vessel segment leakage detection. In our future work, we will extend the current saliency-guided leakage detection model to other vascular diseases, such as diabetic retinopathy. We believe that this innovative framework has the potential to be developed further as a useful tool for fast, accurate and objective assessment of leak in a range of vascular diseases. However, it is still desirable to optimize the parameters of the proposed method in short future, such as the threshold value to generate the binary segmentation of the region of interest from saliency maps, as well as the patch size.

\section{Acknowledgement}

The project was supported by National Basic Research Program of China (2013CB328806), National Natural Science Foundation of China (81430039, 61572076), the Key Projects in the National Science \& Technology Pillar Program (2013BAI01B01), National Hi-Tech Research and Development Program (2015AA043203), and China Postdoctoral Science Foundation Grant (2015M570940), the Key Laboratory of Photoelectronic Imaging Technology and System Beijing Institute of Technology Ministry of Education of China (2016OEIOF03); the Beijing Institute of Technology Research Fund Program for Young Scholars.

\section{References}

1. Chui T, Dubow M, Pinhas A, Shah N, Gan A, Weitz R, et al. Comparison of adaptive optics scanning light ophthalmoscopic fluorescein angiography and offset pinhole imaging. Biomed Opt Express. 2014;5(4):1173-1189.

2. Patton N, Aslam T, MacGillivray T, Pattie A, Deary IJ, Dhillon1 B. Retinal vascular image analysis as a potential screening tool for cerebrovascular disease: a rationale based on homology between cerebral and retinal microvasculatures. J Anat. 2005;206:319-348.

3. Potchen M, Kampondeni S, Seydel K, Birbeck G, Hammond C, Bradley W. Acute brain MRI findings in 120 Malawian children with cerebral malaria: new insights into an ancient disease. Am J Neuroradiol. 2012;33:1740-1746.

4. Beare N, Glover S, Molyneux M. Malarial retinopathy in cerebral malaria. The American Journal of Tropical Medicine and Hygiene. 2009;80:171.

5. Maccormick IJ, Maude R, Beares N, Borooah S, Glover S, Parry D, et al. Grading fluorescein angiograms in malarial retinopathy. Malar J. 2015;14:367.

6. Rabbani H, Allingham M, P M, Cousins S, Farsiu S. Fully automatic segmentation of fluorescein leakage in subjects with diabetic macular edema. Invest Ophthalmol Vis Sci. 2015;56(3):1482-1492. 
7. Martinez-Costa L, Marco P, Ayala G, Ves ED, Domingo J, Simo A. Macular edema computeraided evaluation in ocular vein occlusions. Comput Biomed Res. 1998;31:374384.

8. Phillips R, Spencer T, Ross P, Sharp P, Forrester J. Quantification of diabetic maculopathy by digital imaging of the fundus. Eye. 1991;5:130-137.

9. Saito J, Roxburgh D D Sutton, Ellingford A. A new method of image analysis of fluorescein angiography applied to age-related macular degeneration. Eye. 1995;9:7076.

10. Trucco E, Buchanan CR, Aslam T, Dhillon B. Contextual detection of ischemic regions in ultra-wide-field-of-view retinal fluorescein angiograms. In: Proc. IEEE EMBC; 2007. p. 6739-6742.

11. Tsai CL, Yang CL, Chen SJ, Lin CH K S Chan, Lin WY. Automatic Characterization of Classic Choroidal Neovascularization by Using AdaBoost for Supervised Learning. Invest Ophthalmol Vis Sci. 2011;52:2767-2774.

12. Yuan Y, Wang J, Li B, Meng M. Saliency based Ulcer Detection for Wireless Capsule Endoscopy Diagnosis. IEEE Trans Med Imag. 2015 Apr;.

13. Mahapatra D, Sun Y. MRF-Based Intensity Invariant Elastic Registration of Cardiac Perfusion Images Using Saliency Information. IEEE Trans Biomed Eng. 2011 Apr;58(4):991-1000.

14. Deepak US, Chakravarty A, Sivaswamy J. Visual saliency based bright lesion detection and discrimination in retinal images. In: Proc. IEEE ISBI; 2013. p. 1436-1439.

15. Hou X, Zhang L. Saliency detection: A spectral residual approach. In: Proc. IEEE CVPR; 2007. p. 1-8.

16. Jampani V, Ujjwal, Sivaswamy J, Vaidya V. Assessment of Computational Visual Attention Models on Medical Images. In: Proc. Indian Conf. Comput. Vis. Graph. Imag. Process.; 2012. p. 1-8.

17. Gao D, Mahadevan V, Vasconcelos N. On the plausibility of the discriminant centersurround hypothesis for visual saliency. J Vis. 2008;8(7):1-18.

18. Zhao Y, MacCormick I, Parry D, Beare N, Harding S, Zheng Y. Automated Detection of Vessel Abnormalities on Fluorescein Angiogram in Malarial Retinopathy. Sci Rep. $2015 ; 5: 11154$.

19. Lu Y, Lai Z, Fan Z, Cui J, Zhu Q. Manifold discriminant regression learning for image classification. Neurocomputing. 2015;166:475-486.

20. Yang M, Zhu P, Liu F, Shen L. Joint representation and pattern learning for robust face recognition. Neurocomputing. 2015;168:70-80.

21. Wang X. Learning from big data with uncertainty-editorial. Journal of Intelligent \& Fuzzy Systems. 2015;28(5):2329-2330.

22. Wang XZ, Ashfaq RAR, Fu AM. Fuzziness based sample categorization for classifier performance improvement. Journal of Intelligent \& Fuzzy Systems. 2015;29(3):11851196

23. He YL, Wang XZ, Huang JZ. Fuzzy nonlinear regression analysis using a random weight network. Information Sciences. 2016;364:222-240.

24. He Yl, Liu JN, Hu Yx, Wang Xz. OWA operator based link prediction ensemble for social network. Expert Systems with Applications. 2015;42(1):21-50.

25. Itti L, Koch C, Niebur E. A model of Saliency-Based visual attention for rapid scene analysis. IEEE Trans on Pattern Anal MachIntell. 1998;20:1254-1259.

26. Cheng M, Zhang G, Mitra N, Huang X, Hu S. Global Contrast based Salient Region Detection. In: Proc. IEEE International CVPR; 2011. p. 409-416.

27. Ng W, Yeung D, Firth C, Tsang E, Wang X. Feature selection using localized generalization error for supervised classification problems using RBFNN. Patt Recog. 2018;41:3706-3719.

28. Achanta R, Hemami S, Estrada F, Susstrunk S. Frequency-tuned salient region detection. In: Proceedings of IEEE Conference on Computer Vision and Pattern Recognition (CVPR); 2009. p. 1597-1604.

29. Koch C, Ullman C. Shifts in selective visual attention: towards the underlying neural circuitry. Human Neurbiol. 1985;p. 219-227.

30. Clark J, Ferrier N. Modal control of an attentive vision system. In: Proc. IEEE ICCV; 1988. p. 514-523. 
31. Milanese R. Detecting salient regions in an image: from biological evidence to computer implementation. PhD thesis. 1993;

32. Bruce N, Tsotsos J. Saliency based on information maximization. In: Proc. NIPS. vol. 18; 2006. p. 155-162.

33. Harel J, Koch C, Perona P. Graph-based visual saliency. In: Proc. NIPS; 2006. p. 545-552.

34. Aziz M, Mertsching B. Fast and Robust Generation of Feature Maps for Region-Based Visual Attention. IEEE Trans Image Process. 2008;17:633-644.

35. Wang Z, Li B. A two-stage approach to saliency detection in images. In: Proc. IEEE ICASSP ; 2008. p. 965-968.

36. Guo C, Zhang L. Spatio-temporal saliency detection using phase spectrum of quaternion Fourier transform. In: Proc. IEEE CVPR; 2008. p. 1-8.

37. Cui X, Liu Q, Metaxas D. Temporal spectral residual: fast motion saliency detection. In: Proc. 17th ACM Int. Conf. Multimedia; 2009. p. 617-620.

38. Kienzle W, Wichmann FA, Franz MO, Scholkopf B. A Nonparametric Approach to Bottom-Up Visual Saliency. In: Proc. NIPS; 2006. p. 689-696.

39. Seo H, Milanfar P. Static and space-time visual saliency detection by self-resemblance. J Vis. 2009;9(12):1-27.

40. Goferman S, Zelnik-Manor L, Tal A. Context-Aware Saliency Detection. IEEE Trans Pattern Anal Machine Intell. 2012 Oct;34(10):1915-1926.

41. Gopalakrishnan V, Hu Y, Rajan D. Random walks on graphs for salient object detection in images. IEEE Trans Image Processing. 2011 Apr;24(4):991-1000.

42. Martins P, Carvalho P, Gatta C. Context-aware Features and Robust Image Representations. J Vis Comun Image Represent. 2014;25:339-348.

43. Zhao Y, Liu Y. Using region-based saliency for $3 d$ interest points detection. In: Proc. CAIP; 2013. p. 108-115.

44. Zhao Y, Liu Y, Wu X, Harding SP, Zheng Y. Retinal Vessel Segmentation: An Efficient Graph Cut Approach with Retinex and Local Phase. PLoS ONE. 2015;10:e0122332.

45. Zhao Y, Rada L, Chen K, Harding SP, Zheng Y. Automated Vessel Segmentation Using Infinite Perimeter Active Contour Model with Hybrid Region Information with Application to Retinal Images. IEEE Trans Med Imag. 2015;34:1797-1807.

46. Zhao Y, MacCormick I, Parry D, Leach S, Beare N, Harding S, et al. Automated Detection of Leakage in Fluorescein Angiography Images with Application to Malarial Retinopathy. Sci Rep. 2015;5:10425.

47. Hong X, Chen S, Harris CJ. A Kernel-Based Two-Class Classifier for Imbalanced Data Sets. IEEE Trans Neural Netw. 2007;18:28-41. 
2016-07-26

\section{A compactness based saliency approach for leakages detection in fluorescein angiogram}

\section{Zhao, Yitian}

Springer Verlag

Zhao Y, Su P, Yang J, Zhao Y, Zheng Y, Wang Y, A compactness based saliency approach for leakages detection in fluorescein angiogram, International Journal of Machine Learning and Cybernetics, Vol. 8, Issue 6, December 2017, pp. 1971-1979

http://dx.doi.org/10.1007/s13042-016-0573-4

Downloaded from Cranfield Library Services E-Repository 